\title{
Actitudes y prácticas empleadas por los adolescentes de la ciudad de Somoto en relación al uso de los métodos anticonceptivos y al embarazo
}

\author{
Dra. Anna Planck \\ anna.planck@horizont3000.org \\ Recepcionado: 20 abril, 2013 / Aceptado: 13 de julio, 2013
}

\section{RESUMEN}

El presente estudio sobre los Conocimientos actitudes y Prácticas (CAP) de la población adolescente somoteña ante el uso de anticonceptivos y el embarazo, es de carácter cuantitativo. El objetivo primordial fue analizar este comportamiento y su incidencia en el aumento de embarazo de mujeres menores de 20 años. Se estudiaron las características socio demográficas del grupo, sus conocimientos respecto al embarazo y la prevención del mismo durante la adolescencia, las actitudes que influyen en la reducción o en el aumento de embarazos y las prácticas de protección o riesgo que mantienen los y las adolescentes respecto al embarazo. Se realizó el CAP debido a la elevada incidencia de embarazos adolescentes en el municipio, además no existen otras investigaciones sobre la temática. La muestra fue de 66 adolescentes, ( $50 \%$ de cada sexo). Los principales resultados del estudio mostraron que el 50\% de los adolescentes presenta un nivel satisfactorio de conocimientos sobre la temática. De los varones el $60 \%$ y $39 \%$ de las mujeres ya son activos sexualmente. Existe confusión sobre el uso del anticonceptivo de emergencia "Píldora del día Siguiente" y desconocimiento del doble efecto en el uso del preservativo. La falta de poner en práctica el uso de Métodos Anticonceptivos (MAC) y la confusión del uso adecuado de anticonceptivos expone al grupo objeto de estudio, al aumento de embarazos durante la adolescencia y arriesga su salud sexual y calidad de vida.

Palabras claves: embarazo, adolescente, métodos anticonceptivos, Somoto.

\section{INTRODUCCIÓN}

El embarazo durante la adolescencia constituye un riesgo tanto para las madres como los padres adolescentes, pero sobre todo para sus hijos e hijas.

El embarazo tiene un enorme impacto en la salud de la madre adolescente, especialmente cuando se trata de embarazos no planificados o no deseados (que son la mayoría). La consecuencia en los aspectos psicosociales representa, en la mayoría de los casos, la puerta de entrada o la profundización en el ciclo de la pobreza.

El embarazo en la adolescencia es la principal causa de muerte en las mujeres que tienen edades comprendidas entre 15 y 19 años. Estudios de la UNICEF revelan que cada año 15 millones de adolescentes dan a luz lo que representa el $10 \%$ de los nacimientos registrados anualmente en el mundo. Uno de los países de América Latina y el Caribe que presenta mayor porcentaje de madres adolescentes es Nicaragua, con una tasa de $24 \%$ según datos de la Organización Panamericana de la Salud, (OPS). Los registros del centro de Salud de la ciudad de Somoto y del Sistema Local de Atención

Integral de Salud (SILAIS-Madriz), muestran un porcentaje de embarazo en adolescentes de $19 \%$ en el año 2010, muy por encima de la media mundial que es de $10 \%$ según UNICEF.

Los factores determinantes que influyen en la fecundidad en la adolescencia son múltiples y entre ellos los más descritos son los de origen biológico, los que se relacionan con la conducta sexual, las condiciones dentro de la familias, el desarrollo psicológico, factores socio culturales y accesibilidad a información y anticoncepción. Por ello debe ser de interés constante realizar evaluaciones sobre lo que piensan los adolescentes, los conocimientos que tienen acerca de cada una de los aspectos psicosociales, económicos y culturales en torno al embarazo a temprana edad; los estudios de conocimientos, actitudes y prácticas (CAP) permiten monitorear tanto la información que continuamente se está recibiendo y compararla 
con la conducta del individuo ante una situación objeto de estudio.

\section{MATERIALES Y MÉTODOS}

\section{Tipo de estudio}

Es un estudio CAP o KPC, cuantitativo, sobre los conocimientos, actitudes y prácticas de la población adolescentes de la ciudad de Somoto, de ambos sexos, que inciden en el aumento de embarazos en mujeres menores de 20 años.

Universo y muestra: el universo comprende los y las adolescentes de la ciudad de Somoto, departamento de Madriz, Nicaragua, según INIDE para el año 2010, en este municipio hay 3238 adolescentes. La muestra se conformó por adolescentes de ambos sexos que habitan en la ciudad de Somoto, con un nivel de confianza del $95 \%$ y un error máximo permitido de $5 \%$. Se estimó el tamaño muestral con la fórmula para el cálculo de muestras en estudios descriptivos obteniéndose el valor de 66 adolescentes, 3 de cada barrio que conforman a la ciudad de Somoto (existen 22 barrios). Además, se procuró que dentro de la muestra se correspondiera el $50 \%$ a cada sexo. Los criterios de selección para la muestra fueron: Ser adolescente, (tener entre 15 y 19 años de edad), vivir en la ciudad de Somoto y participar voluntariamente en el estudio.

Es importante señalar que 38 de los y las adolescentes que participaron en la investigación se encontraron en el Instituto Nacional Autónomo de Madriz (escuela segundaria que atiende $85 \%$ de adolescentes de la ciudad), los restantes 28 adolescentes se buscaron en los barrios al azar para cumplir con la muestra de 3 adolescentes por sector.

\section{Técnicas e Instrumentos}

El instrumento empleado fue una encuesta CAP. Este se aplicó de manera individual al o a la adolescente bajo condiciones de privacidad y confidencialidad. La participación era voluntaria. La encuesta posee 23 preguntas entre cerradas y abiertas, las que fueron divididas en 4 secciones según las variables del estudio. Estas fueron las siguientes:

1. Características socio demográficas.

2. Preguntas encaminadas a la búsqueda de información y conocimiento que tienen los adolescentes con relación al embarazo durante esta etapa de desarrollo. Se establecieron escala de valores que permitieron identificar el nivel de conocimiento encontrado.

3. Actitudes frente al embarazo, buscando la disposición psicológica adquirida a través de la propia experiencia de los adolescentes.

4. Prácticas en Sexualidad y Reproducción que inciden en salir o no embarazada, o embarazar o no.

Las actitudes se midieron en base a la escala de Likert, mediante porcentajes, ubicando las opciones en: Totalmente en desacuerdo, en desacuerdo, Indeciso, totalmente de acuerdo y de acuerdo, dándoles un valor numérico en donde los valores altos reflejan una actitud adecuada y los porcentajes bajos indican una actitud inadecuada. Las variables de conocimientos y prácticas fueron medidas a través del número de respuestas correctas y se ubicaron en una escala por nivel de conocimiento.

\section{RESULTADOS}

\section{Conocimientos sobre el embarazo y la prevención del mismo, durante la adolescencia.}

Sobre la duración del ciclo menstrual fisiológico el $65.1 \%$ poseen un conocimiento correcto, mientras que el $34.9 \%$ de adolescentes tienen un conocimiento erróneo respecto a la duración del ciclo menstrual.

En lo que se refiere al período menstrual resulta que $84.8 \%$ de los adolescentes cuentan con conocimiento correcto, (dura entre 3 y 5 días), mientras el $15.2 \%$ refiere que el periodo menstrual fisiológico dilata menos de 3 días o más de 8 días al mes. En cuanto al conocimiento de los días fértiles de la mujer, es llamativo que el $60.6 \%$ de los varones no saben ubicar los días fértiles correctamente, mientras que en el sexo femenino este porcentaje se ubica en un $36.4 \%$. 
Al preguntárseles sobre cómo ocurre el embarazo, el $86.4 \%$ de todos los adolescentes saben que un embarazo ocurre durante una relación coital durante los días fértiles sin uso de un método anticonceptivo (MAC). Sin embargo un $13.6 \%$ creen que un embarazo resulta al tener relaciones sexuales durante la menstruación o unos días antes de que inicia un periodo menstrual.

Respecto al conocimiento sobre la prevención del embarazo el $72.8 \%$ de los adolescentes refieren que un embarazo se previene a través del uso de un MAC, mientras que el $27.2 \%$ mencionan que a través de la abstinencia y al uso de un MAC.

\section{Conocimiento sobre los riesgos que corre una adolescente embarazada y su hijo}

Los adolescentes entrevistados manifestaron que el mayor riesgo que una adolescente embaraza son los problemas médicos (62.1\%). Un 22.6\% consideró que eran problemas familiares y discriminación, mientras que el $6.1 \%$ considero abandono por pareja y otro $6.1 \%$ problemas médicos y fracaso en el estudio y para el futuro. Un $3.1 \%$ otros riesgos.

Los riesgos que corre un niño o una niña de una madre adolescente fueron mencionados en los siguientes porcentajes: $33.3 \%$ maltrato $y$ abandono; $28.9 \%$ problemas médicos (desnutrición, prematuro, enfermedades); $22.7 \%$ maltrato; $9 \%$ abandono, problemas médicos y muerte; $6.1 \%$ muerte.

Las consecuencias futuras para una madre adolescente fueron mencionados en los siguientes porcentajes: $28.9 \%$ abandono por la pareja y por la familia; $22.7 \%$ abandono por pareja; $22.7 \%$ mucha responsabilidad y falta de libertad; $13.6 \%$ fracaso en los estudios; $9 \%$ problemas económicos; $3.1 \%$ problemas psicológicos.

\section{Conocimientos sobre los Métodos de Anticoncepción}

El $50 \%$ de los y las adolescentes conocen la pastilla, el condón y la inyección, $25.8 \%$ mencionan la pastilla, el condón, la inyección y la pastilla para la mañana siguiente, solamente la pastilla y el condón conocen el $17.6 \%$, otro $7.6 \%$ conocen la pastilla, el condón la inyección y el dispositivo intrauterino y $9 \%$ mencionan otras combinaciones.

Respecto al momento adecuado para iniciar con el uso de un MAC posee el $65.2 \%$ de los y las adolescentes un conocimiento adecuado mientras un $34.8 \%$ tienen conocimientos incorrectos (nunca en menores de 16 años y al cumplir los 16 años).

Para acceder a un MAC los adolescentes de ambos sexos refieren los siguientes lugares: $33.3 \%$ Centro de Salud y Hospital; 28.8\% Centro de Salud, Hospital y Farmacia; $21.2 \%$ Centro de salud y organización no gubernamental; $10.6 \%$ venta $y$ farmacia; $6.1 \%$ otros lugares.

La ventaja más mencionada sobre el uso del preservativo que refieren conocer los y las adolescentes fue la prevención del embarazo y de infecciones de transmisión sexual (en un 40.9\%), mientras que la desventaja más mencionada fue que no evita las infecciones de transmisión sexual en cien por ciento (36.4\%).

La ventaja de la pastilla que más refieren conocer los y las adolescentes fue su eficacia contra el embarazo (60.6\%), mientras que las principales desventajas de la pastilla que conocen adolescentes de ambos sexos son los problemas físicos para la mujer mencionándolos un $27.2 \%$, además del hecho de que la pastilla no evita ITS lo refieren $21.2 \%$.

Los y las adolescentes en un $50 \%$ mencionan que la principal ventaja de DIU (dispositivo uterino) es su eficacia contra el embarazo. Las adolescentes refieren, además, que otra ventaja es su uso local $7.6 \%$ y el $42.4 \%$ no conocen ninguna ventaja de este MAC.

Respecto a las desventajas del DIU un $24.2 \%$ de los adolescentes de ambos sexos mencionan que se puede "encarnar" o desviar, $12.1 \%$ refiere que es incómodo y causa dolor, $6.1 \%$ que no protege contra ITS, $45.4 \%$ no conocen ninguna desventaja acerca de este método anticonceptivo y $12.1 \%$ mencionan otras desventajas. 
Los y las adolescentes refieren que la principal ventaja de la inyección es su eficacia contra el embarazo (53\%). Respecto a las desventajas de la inyección, $48.4 \%$ mencionan problemas físicos en la mujer (mancha la cara, engorda, descontrola el ciclo).

\section{Actitudes relacionadas al embarazo y la prevención del mismo, durante la adolescencia}

En relación a la opinión de los adolescentes varones sobre tener varias parejas sexuales al mismo tiempo, resulta que el $12.1 \%$ opina que definitivamente que sí y probablemente que si $27.3 \%$, mientras el $6.1 \%$ están indecisos y el $54.5 \%$ opinan que probablemente o definitivamente no tendrían varias parejas sexuales simultáneas.

Las adolescentes mujeres tienen las siguientes opiniones respecto a tener varias parejas sexuales simultáneas: El $6.1 \%$ opinan que definitivamente, $12.1 \%$ probablemente, $15.2 \%$ están indecisas, 18.2 refieren que probablemente no y $48.4 \%$ definitivamente no tendrían múltiples parejas sexuales.

La opinión de los varones respecto al uso de un MAC en relaciones sexuales futuras la encuesta refleja lo siguiente: el $57.5 \%$ opina que definitivamente que sí, el $27.3 \%$ probablemente que sí, 9.1\% están indecisos, $6.1 \%$ dicen que probablemente que no y ninguno opina que definitivamente que no. Mientras que las adolescentes mujeres opinan sobre el potencial uso de un MAC en una relación sexual futura los siguiente: el $63.6 \%$ opina que definitivamente que sí; el 30.3\% probablemente que sí, $6.1 \%$ están indecisas, y ninguna opina que probablemente o definitivamente que no.

Respecto a la actitud de los adolescentes varones en usar en cada relación sexual un MAC, se obtiene la siguientes opiniones: el $54.5 \%$ definitivamente que sí, el $30.3 \%$ probablemente que sí, $9.1 \%$ están indecisos, $6.1 \%$ dicen que probablemente que no y ninguno refiere definitivamente que no. En el caso de las adolescentes mujeres manifiestan en un 63.6\% que definitivamente que sí usarían un MAC en sus relaciones sexuales, $33.3 \%$ probablemente que sí, mientras que el $3 \%$ se mostró indecisas.

Sobre la actitud que tomarían los adolescentes varones teniendo relación sexual sin MAC, si la pareja se lo pediría se obtuvo las siguientes opiniones: el $12.1 \%$ definitivamente que sí, $12.1 \%$ probablemente que sí, el $27.3 \%$ está indeciso, otro $30.3 \%$ refiere que probablemente que no y un $18.1 \%$ opinan que definitivamente que no. Sobre la actitud que tomarían las adolescentes mujeres si tendrían relación sexual sin MAC, si la pareja se lo pidiera se obtuvo las siguientes opiniones: ninguna dice que definitivamente que sí, 3\% probablemente que sí, $15.2 \%$ están indecisas, el $18.2 \%$ refieren que probablemente que no y un $63.6 \%$ opinan que definitivamente que no.

Respecto a la actitud que optaran los adolescentes varones, de dejar a su pareja si esta saliera embarazada, resultó lo siguiente: el $18.2 \%$ opinan que definitivamente que sí, $21.2 \%$ probablemente que sí, $15.2 \%$ están indecisos, $21.2 \%$ probablemente que no y $24.2 \%$ definitivamente que no. Mientras que las mujeres al preguntárseles si abortarían en caso de estar embarazada durante la adolescencia, respondieron: el $6.1 \%$ opinan que definitivamente que sí, $12.1 \%$ probablemente que sí, $9.1 \%$ están indecisas, $18.2 \%$ probablemente que no y $54.5 \%$ definitivamente que no.

Acerca de la actitud que toman los adolescentes varones si tuvieran relaciones sexuales coitales sin MAC para agradar a su pareja opinan como sigue: el $12.1 \%$ opinan que definitivamente que sí, $15.2 \%$ probablemente que sí, $27.3 \%$ están indecisos, $27.3 \%$ probablemente que no y $18.1 \%$ definitivamente que no. La actitud de las adolescentes mujeres si tuvieran relaciones sexuales coitales sin MAC para agradar a su pareja resultó de la siguiente manera: el 3\% menciona que definitivamente que sí, $6.1 \%$ adolescentes afirman que probablemente sí, $15.2 \%$ son indecisas, $18.2 \%$ refieren que probablemente no lo harían y $57.5 \%$ dicen definitivamente que no. 


\section{Prácticas sobre el embarazo y la prevención del mismo, durante la adolescencia}

En relación al inicio de la vida sexual los adolescentes varones muestran la siguiente práctica: $60.6 \%$ ya iniciaron vida sexual activa, $39.4 \%$ no han iniciado vida sexual activa. Mientras que las adolescentes muestra que $39.3 \%$ ya han comenzado a tener relaciones sexuales coitales, mientras que el $69.7 \%$ refieren que aún no han iniciado.

Al indagar sobre la edad de inicio de la vida sexual activa contestaron los varones adolescentes, que el $40 \%$ comenzó a tener relaciones sexuales a los 16 años cumplidos, $25 \%$ con la edad de 17 años, $15 \%$ a los 18 años, $10 \%$ a los 15 años y $10 \%$ a la edad de 14 años. En el caso de las mujeres resulta que $30.7 \%$ comenzaron con 18 años y $23.1 \%$ respectivos para las edades de 16 y 17 años y $15.4 \%$ a los 14 años, $7.7 \%$ a los 14 años.

También se indagó a los adolescentes sobre el número de parejas sexuales que han tenido desde que iniciaron vida sexual activa: los varones muestran al respecto las siguientes prácticas: $65 \%$ han tenido una sola pareja, $25 \%$ refieren haber tenido dos parejas sexuales y $10 \%$ tuvieron cuatro parejas sexuales. Las adolescentes mujeres tienen las siguientes prácticas relacionados al número de parejas sexuales: $69.2 \%$ una sola pareja, $15.4 \%$ dos parejas y otros $15.4 \%$ no quiso manifestarse al respecto.

Respecto al uso actual de un MAC en los adolescentes sexualmente activos, resulta que: en los varones el $75 \%$ usa un MAC y $25 \%$ no usan, de las adolescentes mujeres un $61.5 \%$ si usa un MAC y $38.5 \%$ no lo usan.

De los adolescentes sexualmente activos un $45 \%$ ya ha engendrado un hijo/a, mientras el $55 \%$ no. De las adolescentes mujeres el $38.5 \%$ no se ha embarazado, mientras el $61.5 \%$ ha estado embarazada. De los adolescentes varones que han causado un embarazo, $33.3 \%$ (3) son padres adolescentes y de las adolescentes que han estado embarazadas $37.5 \%$ (3) son madres.

\section{DISCUSIÓN}

\section{Conocimientos sobre el embarazo y la prevención del mismo, durante la adolescencia}

Solamente la mitad del grupo estudiado alcanza un nivel satisfactorio de conocimientos sobre el ciclo menstrual, el período menstrual y la ubicación de los días fértiles. Es evidente entonces que existe carencia de información especialmente en torno a la localización de los días fértiles en un ciclo menstrual fisiológico.

La gran mayoría sabe cómo ocurre un embarazo y todos indican que los métodos anticonceptivos son para prevenir un embarazo y casi la mitad de adolescentes refiere adicionalmente a la abstinencia sexual como prevención. Respecto a los riesgos a los cuales se afronta una adolescente embarazada y un niño o niña de una madre adolescente existe una amplia gama de conocimientos, resaltando principalmente los problemas médicos e incluso la muerte. Sobre las consecuencias psico-sociales tanto para la embarazada adolescente, madre adolescente y niño o niña de ella, el grupo de los estudiados está bien informado, mencionando múltiples situaciones, de las cuales sobresalen los problemas familiares, el abandono, la discriminación social y el fracaso en los estudios. Llama la atención que la mayoría de los y las adolescentes no visibilizan las dificultades económicas a las cuales se afronta una madre adolescente. Todos los adolescentes conocen varios métodos anticonceptivos, entre estos la gran mayoría conoce tres o más métodos.

Existe una marcada confusión respecto a la "Pastilla para la mañana siguiente" (levonorgestrel), ya que cerca la mitad de los encuestados la mencionan como un MAC de uso rutinario. Preocupa que solamente muy pocos adolescentes (19\%) refieran como fuente de información a los centros educativos, dato que muestra la debilidad generalizada en el abordaje del tema en las escuelas públicas del lugar. Sin embrago muchos estudios como por ejemplo: "La educación sexual ayuda a preparar a los jóvenes, $\mathrm{FHI}$, Thescience of improvinglive, 2000", llegan a la conclusión de que la educación sexual no da lugar a que adolescentes 
tengan relaciones sexuales precoces o que éstas sean más frecuentes, más bien puede favorecer el retraso para su primera relación sexual o, si ya están sexualmente activos, que usen la anticoncepción.

Alarmante es la casi ausencia (2\%) de padres y madres respecto a la información sobre salud sexual, indicando que la comunicación alrededor de la temática está muy limitada en las familias. Este resultado se corrobora en muchas investigaciones que afirman que la familia debe ser la base de una buena educación sexual brindando una precisa información sexual, libre de tabúes y mitos comunes que rodean a todo lo que tiene que ver con la sexualidad. Existe un desconocimiento marcado respecto al doble efecto del preservativo, ya que solamente $41 \%$ de los y las adolescentes mencionaron su efectividad anticonceptiva $y$ prevención contra infecciones de transmisión sexual.

Notablemente también la cantidad de adolescentes que no saben mencionar ninguna ventaja respecto al condón, especialmente en las adolescentes mujeres.

Sin embargo mencionan una variedad de desventajas respecto a este MAC, de los cuales la mayoría indican su uso inadecuado, (causa dolor, y es incómoda). La eficacia contraceptiva de los métodos anticonceptivos hormonales $y$ del dispositivo intrauterino es la principal ventaja que conocen los y las adolescentes.

La desventaja principal de estos métodos es la no protección contra infecciones de transmisión sexual es muy poco conocido por adolescentes de Somoto, solo el $25 \%$ la identifican. Las adolescentes mujeres son las que tienen un poco más conocimientos sobre los MAC que los varones de su misma edad, especialmente sobre ventajas y desventajas de los MAC que son utilizados por la mujer. EI MAC menos conocidos por los adolescentes es el dispositivo intrauterino (DIU), lo que se correlaciona con el hecho que este no es un MAC que se promueve en este grupo etario. Existen aún creencias erróneas relacionadas a los anticonceptivos, especialmente referentes al DIU y a la inyección. Los dispositivos intrauterinos se desvían o "encarnan", la inyección envenena la sangre, la pastilla provoca esterilidad.

Investigaciones han identificado tres tipos de barreras que interfieren con el acceso de adolescentes a información sobre salud sexual y acceso a métodos anticonceptivos, estas se relacionan con las características de las instalaciones, el diseño de los programas y el trato de los proveedores, demostrado en Mejorando el Acceso de Jóvenes a Métodos Anticonceptivos en América Latina, AdvocatesforYouth, (2001).

Es significativo que más de la mitad de los adolescentes somoteños mencionan una unidad de salud estatal del Ministerio de Salud como primer opción para acceder a información relacionado al embarazo y su prevención y a un MAC. La farmacia representa la segunda opción.

\section{Actitudes sobre el embarazo y la prevención del mismo, durante la adolescencia}

Como actitudes favorables relacionado a la prevención del embarazo es la afirmación de adolescentes de ambos sexos respecto al uso consecuente de un método anticonceptivo en cada relación sexual coital.

A esto se contrapone sin embargo, la opinión de una tercera parte de adolescentes que vincula el inicio del uso de una MAC con una edad específico y refiriendo que no se puede usar un MAC en menores de 16 años. Práctica que exponen a adolescentes al riesgo de embarazar o quedar embarazado es el hecho de tener varias parejas sexuales simultáneas, actitud que exteriorizan adolescentes varones, (39\%).

La actitud de adolescentes de tener relaciones sexuales sin MAC por presión de la pareja o para agradar a la pareja, resultó igualmente en los varones desfavorable, aunque algunos adolescentes de sexo masculino, tienden a sobre declarar su conducta sexual, al contrario que los de sexo femenino. (Barella, 2002). Las adolescentes mujeres tienen una actitud favorable al respecto, manifestando en su mayoría que no se dejarían presionar por su pareja y que no tendrían relaciones sexuales sin MAC para agradar a su 
pareja. Si se suma el hecho de que cerca de la mitad de los adolescentes dejarían a su pareja si esta saldría embarazada a las actitudes que inciden en el aumento del embarazo en la adolescencia, la situación alrededor de la temática se torna preocupante.

\section{Prácticas sobre el embarazo y la prevención del mismo, durante la adolescencia}

Cerca de dos tercios de los varones adolescentes y un tercio de las adolescentes mujeres ya iniciaron vida sexual activa, lo que corresponde a estadísticas nacionales y mundiales, que muestran, que entre adolescentes prevalece un conocimiento teórico sobre métodos anticonceptivos para evitar el embarazo, pero en la práctica muchos no lo usan, resultados descritos en el estudio de Pérez (2008) quien indica que el inició de vida sexual activa entre los 15 y 17 años para ambos sexos, correspondiendo esta situación a la adolescencia media. Un tercio de los adolescentes varones indica haber tenido más de una pareja sexual en el período del estudio, y a pesar de estar sexualmente activo, $25 \%$ de los adolescentes varones y $20 \%$ de las adolescentes mujeres no han utilizado un MAC, prácticas que indudablemente inciden directamente en el aumento de embarazos adolescentes y probablemente no deseados. Las prácticas desfavorables se corroboran entre los y las adolescentes con vida sexual activa, al analizar el dato respectivo al haber engendrado un hijo o haber estado ya embarazada. Reafirman las prácticas desfavorables el hecho de que los varones que han engendrado un hijo solamente un tercio son padres y de las embarazadas adolescentes solamente una cuarta parte son madres.

\section{CONCLUSIONES}

Los y las adolescentes sometidos a estudio se encuentran en su mayoría en la adolescencia media, masculino y femenino en igual distribución, con un nivel de estudio de segundaria, aunque deberían estar solteros/as, existe una cuarta parte de ellos que ya mantienen una unión, bajo el núcleo familiar de sus padres.
Solamente el $50 \%$ de los y las adolescentes en estudio tienen un nivel satisfactorio de conocimientos sobre el ciclo menstrual, el embarazo, el uso de métodos anticonceptivos, reconociendo a la abstinencia como la mejor manera de evitar a un embarazo, pero muestran grave confusión al mencionar la PPMS como un método rutinario de anticoncepción, desconocen el doble efecto protector del condón y persisten creencias erróneas sobre los MAC.

El no usar una método anticonceptivo en la relación sexual para agradar a la pareja o por presión de la pareja, vincular el inicio de uso de un método a la edad después de los 16 años, así como el dejar a la pareja si saliese embarazada y abortar, son aspectos desfavorables de actitud que resaltaron en los adolescentes en este estudio y favorecen el incremento de los embarazos en la adolescencia.

Más de la mitad de los varones adolescentes iniciaron vida sexual activa en la adolescencia media, de las adolescentes mujeres un tercio están con vida sexual activa y aunque permanece el conocimiento sobre la prevención del embarazo no lo practican. Un tercio de todos los adolescentes con vida sexual activa no usa métodos anticonceptivos actualmente, más de la mitad de los adolescentes sexualmente activos han estado embarazadas o han engendrado y de estos la mayoría no se responsabiliza en la práctica de la paternidad o maternidad.

\section{BIBLIOGRAFÍA}

Advocats for Youth. (2005). Mejorando el acceso de los jóvenes a métodos anticonceptivos en América Latina. Obtenido de http://www.

advocatesforyouth.org/publications

Barella Balboa, J. L. (2002). Conocimientos y actitudes sobre sexualidad de los adolescentes de nuestro entorno. Obtenido de http://www.samfyc.es

OPS, Boletín de la Organización Mundial de la Salud, Embarazo en adolescentes: Un problema culturalmente complejo, Enero 2009 
http://www.who.int/bulletin/volumes/87/6/09020609/es/index.html

UNICEF, Embarazo en Adolescentes, Supervivencia Infantil. Estado Mundial de la Infancia. / UNICEF, 2006

http://www.unicef.org/republicadominicana/he alth childhood 10191.htm

Maternidad adolescente en América Latina y el Caribe, Boletín de la infancia y adolescencia sobre el avance de los objetivos de desarrollo del Milenio, 2007 http://www.eclac.org/dds/noticias/desafios/1/2 7871/desafios 4 esp Final.pdf

World population, Youth, Population pyramid and List of countries by median age, 2006 http://en.wikipedia.org/wiki/World population \#Youth

OMS Revista de Posgrado de la Cátedra Vla Medicina No 107 - Agosto/2001 Embarazo en Adolescencia, Prof. Dr. Juan R. Issler

UNFPA Embarazo en la Adolescencia, Porcentaje de embarazos en la adolescencia en países del
Centro América y el Caribe, 2001 http://www.unfpanic.

Redes de Solidaridad Observatorio de Igualdad de Género de América Latina y el Caribe, Informe Anual 2011. Managua, Nicaragua rsolidaridad@ gmail.com

Martínez, Oscar Soto Embarazo en la adolescencia y conocimientos sobre sexualidad, 2003

MINED Educación de la Sexualidad: Guía básica de consulta para docentes 2011 www.nicaraguaeduca.edu.ni

MINISTERO DE SALUD Dirección Primer Nivel de Atención, Estrategia y Lineamientos básicos para la Prevención del Embarazo en Adolescencia, 2003 http://www.puntos.org.ni/

Pérez Hernández, F. (2008). Adolescencia e inicio precoz de las relaciones sexuales. Obtenido de http://bvs.sld.cu/revistas/gme/pub/vol.1.(2) 01 Lp1.html 